\title{
Enhancing the Incidental Pipeline in Genomic Sequencing
}

\author{
B.D. Solomon
}

Division of Medical Genomics, Inova Translational Medicine Institute, Inova Health System, and Department of Pediatrics, Inova Children's Hospital, Falls Church, Va., USA

\begin{abstract}
Key Words
Exome $\cdot$ Genome $\cdot$ Genomic sequencing $\cdot$ Incidental medical information $\cdot$ Incidental pipeline
\end{abstract}

Recent advances have increased the accuracy, affordability, and availability of high-throughput sequencing. Genomic sequencing (including both whole-exome and whole-genome sequencing) is beginning to be explored in the practice of prospective medicine. Currently, however, the most common use involves trying to explain a patient's presentation by identifying a causative genetic variant. Here, the traditional clinical evaluation often progresses towards genomics research. For example, a patient with a disorder of unknown etiology may exhaust the standard clinical work-up, typically involving examining specific genes of interest and/or performing testing to look for structural genomic alterations. If no genetic explanation has been found, the patient may undergo genomic sequencing, sometimes on a research basis. Of note, the distinction between 'clinical sequencing' and 'research-based sequencing' is sometimes blurred, especially in the context of rare diseases. For example, some research labs conduct standard sequencing of known target genes for a certain disorder and will return these results relatively efficiently (whether positive or negative); if nothing is found, these same labs will continue to use the samples to investigate novel targets on a more classic research basis. Again, if a genetic explanation is then found and proven, this would then be returned to the research participants, even if long after initial participation.

In addition to discoveries that may end the diagnostic odyssey, many genomic endeavors include provisions aimed at managing 'incidental medical information' [Solomon et al., 2012; Green et al., 2013]. In this context, incidental medical information includes genomic data that may not be directly relevant to the primary question (what is the reason for the patient's presentation), but that may nonetheless have health implications [Berg et al., 2011, 2013; Burke et al., 2013; Green et al., 2013]. A significant number of variants of potential medical significance are contained in every genome [Xue et al., 2012]. The optimal management of these variants is a critical and controversial area, and much work is required to continue to establish relevant guidelines and approaches [Beskow et al., 2010; Berg et al., 2013; Burke et al., 2013; Green et al., 2013; Yu et al., 2013].

Setting aside the question of what constitutes medically significant incidental genomic data allows a focus on a different but nonetheless important question: what are key considerations in establishing a pipeline for incidental medical information management? Here, based upon our experience, we describe 8 relevant general considerations that may be of use whether genomic sequencing is performed on a purely clinical basis, as part of a research study, or, as is frequent, as a combination of the 2 .

\section{KARGER}

E-Mail karger@karger.com

www.karger.com/msy
(C) 2014 S. Karger AG, Basel

$1661-8769 / 14 / 0052-0047 \$ 39.50 / 0$
Benjamin D. Solomon

Division of Medical Genomics, Inova Translational Medicine Institute Claude Moore Building, 2nd Floor

3300 Gallows Road, Falls Church, VA 22042 (USA)

E-Mail benjamin.solomon@inova.org 


\section{Algorithms to Help Bin Variants}

Every human genome contains a diverse group of medically relevant variants, and manual analysis of each variant is neither feasible nor optimal [Xue et al., 2012; Dorschner et al., 2013]. To help provide efficient and evidence-based variant consideration, automated algorithms may be built into the analysis pipeline. By highlighting certain genes, general types of variants, or specific variants, these algorithms can help focus attention on variants that warrant further (manual) inspection [Berg et al., 2011, 2013; Dorschner et al., 2013].

How the 'bar is set' in terms of which genes and what types of variants deserve primary attention will depend upon context-specific factors and is not the main subject of this article (see below). Beyond general tenets that medically actionable variants will be managed appropriately, determining how variants will be categorized prior to sequencing analysis is anticipated to benefit patients, sequencing facilities, and the involved health care practitioner [Berg et al., 2011; Solomon et al., 2012; Berg et al., 2013].

\section{The Need for Flexibility}

Due to our evolving understanding of genes and their associated disorders, the algorithms described above cannot remain static: there must be capability to revise these algorithms to keep pace with increasing genomic knowledge and to re-evaluate genomic data as indicated [Berg et al., 2013; Solomon et al., 2013]. This becomes especially important when genomic data is intended to be incorporated into ongoing healthcare [Yu et al., 2013]. Further, even with the most carefully-wrought algorithms, challenges to interpretation (gray zones) can and should remain, and consideration of some variants will require additional scrutiny [Beskow et al., 2010; Solomon et al., 2012; Dorschner et al., 2013].

Here, the context of sequencing is paramount, and even with further progress in genomic interpretation, a 'one size fits all' approach cannot suffice [Beskow et al., 2010]. Determining how best to manage results will depend on factors such as the reason for sequencing and on specific clinical characteristics of the individual. For example, the management of a high risk cancer mutation may vary considerably if found in a child participating in a study on the use of genomic sequencing in healthy children versus an adult with a personal or family history of cancer.

\section{Availability and Involvement of the Genomicist}

Experts with relevant genomic experience and interest should be available prior to, during, and after genomic sequencing. The vast majority of clinicians, while open to using genomic information and eager to provide the best care, may lack the necessary time, training, and resources to best incorporate genomics into clinical practice [Nippert et al., 2011; Kaye, 2012]. Adding to practitioner and system responsibilities, even when intended to enhance patient care, may be counter-productive.

To assist with the incorporation of genomic data into healthcare, knowledgeable 'genomicists' (often primarily associated with the sequencing or research team, and ideally consisting of clinical geneticists and genetic counselors) should be involved to help answer questions about the generation, interpretation, and use of genomic information, direct patients and practitioners to further resources such as suitable specialists, clinical studies, and the appropriate reference or CLIA (Clinical Laboratory Improvement Amendments) certified molecular laboratories for further testing and validation, and otherwise ensure appropriate care. This comprehensive genomics approach may frequently extend to in-person meetings with the patient and family.

\section{The Need for Individual Gene/Condition Experts}

Beyond the genomicist, individual disease specific experts are often necessary to help consider a specific variant or phenotype. First, in terms of genotypic analysis, the interpretation of any given variant in terms of potential pathogenicity can be challenging [Johnston and Biesecker, 2013]. A high-fidelity animal model to test the effect of a variant is generally neither possible nor practical. Software-based analysis tools based on factors such as variant type, location, and evolutionary conservation of the affected residue and region can be helpful [Ramensky et al., 2002; Choi et al., 2012] but cannot compete with intimate knowledge of a gene or condition that can only be supplied by an experienced expert in a given disease [Ball et al., 2012; Solomon et al., 2013].

Second, related to phenotypic considerations, experts in the clinical aspects of (often rare) genetic conditions can often supply key information such as awareness of certain phenotypic features that may aid in clinical evaluation or nuances of diagnosis and management that can help determine how genetic information is assessed. In addition to literature searches to find experts, databases
Mol Syndromol 2014;5:47-50 DOI: $10.1159 / 000357929$
Solomon 
like the Genetic Testing Registry (http://www.ncbi.nlm. nih.gov/gtr/) or the National Organization for Rare Disorders (http://www.rarediseases.org/) contain links to resources including individuals who are often willing to provide pertinent discussion.

\section{Ongoing Contact with Sequenced Individuals}

As described above, analysis algorithms must be designed to change with the pace of genetic discovery. However, the most rapidly evolving algorithms will not be helpful if the benefits cannot be returned to patients [Johnston et al., 2012; $\mathrm{Ng}$ et al., 2013]. The channel for continued contact should stay open to allow for ongoing medical involvement and communication. Sequenced individuals should remain aware that genomic interpretation is not a single test that can be completed, and any involved health care practitioner (such as a primary care practitioner) should also be aware that new information may be added or existing information reinterpreted [Ball et al., 2012].

Further, as described below, additional samples may be necessary for validation. In order to keep potentially beneficial information from being trapped by logistic hurdles, contact with the sequenced individual should remain available.

\section{Consideration of Familial Factors}

Identifying the explanation for a patient's phenotype can be greatly enhanced by simultaneous familial sequencing. For example, de novo and bi-allelic mutations can be quickly identified, and variants of potential interest may be of less interest if observed in an unaffected relative [Sincan et al., 2012].

With incidental medical data, the presence of familial data can be similarly helpful in providing evidence for or against disease association. However, incidental medical information identified in every sequenced individual may require consideration similar to that in the proband. A person who undergoes sequencing to help understand, for example, the cause of their child's condition, must be aware of the ramifications relating to incidental medical information. These ramifications include genetic information that may be important for their own health as well as that of additional relatives who may share the same genetic variant [Johnston et al., 2012; Ng et al., 2013].

Enhancing Incidental Pipeline

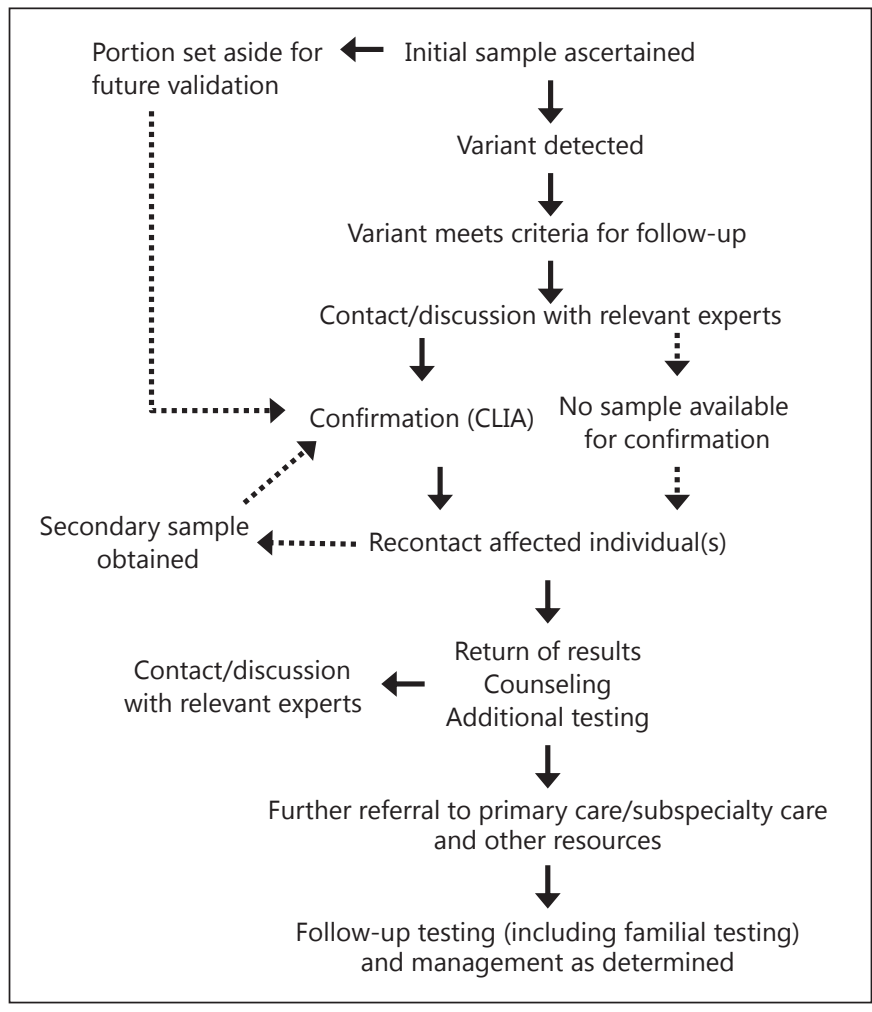

Fig. 1. The incidental pipeline. Through genomic sequencing, a variant of interest is detected; if the variant meets criteria for further action and the sequencing data are considered high quality (it is important to reemphasize that these critical 'ifs' fall outside the scope of this article), initial discussion with specific experts for input related to genotypic and/or phenotypic interpretation may be helpful. If the variant is then still deemed worthy of further action, confirmation is required. This confirmation would follow the necessary regulations to allow the generation of a formal report. As shown (dotted lines), performing confirmation may require obtaining a second patient sample, though this is not ideal in terms of efficient data management. The confirmed result is returned to the individual, ideally in person to allow for counseling and discussion of potential additional tests or interventions. Depending on further information garnered through discussion with the patient, recontacting relevant experts may be helpful, as the availability of more clinical information (such as additional family history) may help determine the optimal course. Finally, further referral can be made to medical practitioners (as well as to other resources such as appropriate patient networks); these practitioners can help tailor further care to the specific patient.

\section{Sample Handling and Confirmation}

While the accuracy of genomic sequencing is improving, validation of any important result is necessary. Challenges that can arise include lack of an available sample for confirmation, questionable sample quality, unexpect- 
ed and sometimes unexplained difficulties with sequencing, and the potential for sample mix-up or contamination. In such instances, progress towards validating and returning a medically meaningful variant may cease. With forethought, 3 general options are available to help minimize barriers: first, simultaneous orthogonal sequencing may help reduce the need for separate validation. Even with orthogonal sequencing, separate validation may be necessary, such as when results from orthogonal platforms are unclear. Second, a portion of the sample may be initially set aside for variant confirmation (especially when establishing cell lines is not possible or when a condition may impact survival). Third, individuals may agree at initial assessment to be recontacted to submit samples for confirmation.

Results must be validated before they are provided to individuals, but the best interpretation of relevant rules and regulations may not also be entirely obvious. In the United States for example, there is disagreement about the exact interpretation of CLIA regulations in the context of large-scale genetic/genomic research [Burke et al., 2013; Green et al., 2013]. Some research laboratories have the capacity to perform in-house CLIA certification of any genetic/genomic variant. Separately, commercial companies exist that can provide confirmation for any research-generated variant and can send sample collection kits to indi- viduals to obtain new samples. The fee for such confirmation is typically a fraction of the cost of commercial sequencing for even one gene, and with appropriate logistical considerations, may be paid by insurance.

\section{Establishing Realistic Expectations}

In summary, the advent of genomic sequencing has generated a great deal of attention. Sequenced individuals as well as many health care practitioners may expect that genomic sequencing will be able to deliver results as quickly and unequivocally as many other medical tests. An underlying theme running through this entire manuscript is that clinically-related genomic sequencing is in its early days, with best practices still being established, and that management of genomic data might best be viewed as part of a process, much like the longitudinal management of a person's overall health [Ball et al., 2012; Burke et al., 2013; Green et al., 2013; Yu et al., 2013].

\section{Acknowledgments}

The author is grateful to Dr. John E. Niederhuber for his helpful comments.

\section{References}

Ball MP, Thakuria JV, Zaranek AW, Clegg T, Rosenbaum AM, et al: A public resource facilitating clinical use of genomes. Proc Natl Acad Sci USA 109:11920-11927 (2012).

-Berg JS, Khoury MJ, Evans JP: Deploying whole genome sequencing in clinical practice and public health: meeting the challenge one bin at a time. Genet Med 13:499-504 (2011).

Berg JS, Adams M, Nassar N, Bizon C, Lee K, et al: An informatics approach to analyzing the incidentalome. Genet Med 15:36-44 (2013).

Beskow LM, Burke W: Offering individual genetic research results: context matters. Sci Transl Med 2:38cm20 (2010).

-Burke W, Matheny Antommaria AH, Bennett R, Botkin J, Clayton EW, et al: Recommendations for returning genomic incidental findings? We need to talk! Genet Med (2013) [Epub ahead of print].

Choi Y, Sims GE, Murphy S, Miller JR, Chan AP: Predicting the functional effect of amino acid substitutions and indels. PLoS One 7:e46688 (2012).

- Dorschner MO, Amendola LM, Turner EH, Robertson PD, Shirts BH, et al. Actionable, pathogenic incidental findings in 1,000 participants' exomes. Am J Hum Genet 93:631-640 (2013).
Green RC, Berg JS, Grody Kalia SS, Korf BR, et al: ACMG recommendations for reporting of incidental findings in clinical exome and genome sequencing. Genet Med 15:565-574 (2013).

Johnston JJ, Biesecker LG: Databases of genomic variation and phenotypes: existing resources and future needs. Hum Mol Genet 22:R27-31 (2013).

Johnston JJ, Rubinstein WS, Facio FM, Ng D, Singh LN, et al: Secondary variants in individuals undergoing exome sequencing: screening of 572 individuals identifies highpenetrance mutations in cancer-susceptibility genes. Am J Hum Genet 91:97-108 (2012).

Kaye CI: Genetic service delivery: infrastructure, assessment and information. Public Health Genomics 15:164-171 (2012).

Ng D, Johnston JJ, Teer JK, Singh LN, Peller LC, et al: Interpreting secondary cardiac disease variants in an exome cohort. Circ Cardiovasc Genet 6:337-346 (2013).

Nippert I, Harris HJ, Julian-Reynier C, Kristoffersson U, Ten Kate LP, et al: Confidence of primary care physicians in their ability to carry out basic medical genetic tasks-a European survey in five countries-Part 1. J Community Genet 2:1-11 (2011).
Ramensky V, Bork P, Sunyaev S: Human nonsynonymous SNPs: server and survey. Nucleic Acids Res 30:3894-3900 (2002).

-Sincan M, Simeonov DR, Adams D, Markello TC, Pierson TM, et al: VAR-MD: a tool to analyze whole exome-genome variants in small human pedigrees with Mendelian inheritance. Hum Mutat 33:593-598 (2012).

- Solomon BD, Hadley DW, Pineda-Alvarez DE, Hansen NF, Kamat A, et al: Incidental medical information in whole-exome sequencing. Pediatrics 129:e1605-1611 (2012).

- Solomon BD, Nguyen AD, Bear KA, Wolfsberg TG: Clinical genomic database. Proc Natl Acad Sci USA 110:9851-9855 (2013).

Xue Y, Chen Y, Ayub Q, Huang N, Ball EV, et al: Deleterious- and disease-allele prevalence in healthy individuals: insights from current predictions, mutation databases, and population-scale resequencing. Am J Hum Genet 91: 1022-1032 (2012).

Yu JH, Jamal SM, Tabor HK, Bamshad MJ: Selfguided management of exome and whole-genome sequencing results: changing the results return model. Genet Med 15:684-690 (2013). 